problems, perhaps because of the relatively small muscle mass involved in these injuries.

\footnotetext{
1 Shaw, R. S., fournal of Bone and foint Surgery, 1959, 41A, 665.

2 Malt, R .A., Remensnyder, J. P., and Harris, W. H., Annals of Surgery 1972, 176, 334

3 Chinese Medical fournal, 1973, 1, 1.

Kleinert, H. E., Kasdan, M. L., and Romero, J. L., fournal of Bone and foint Surgery, 1903, 45A, 788.
}

\section{Glomus Tumours}

A glomus unit consists of an arteriole, a venule, and an anastomotic vessel that directly connects the two. It is lined by endothelium and surrounded by smooth muscle cells, interspersed among which are the cuboidal, epithelium-like glomus cells from which the glomus tumour, or glomangioma, arises. ${ }^{1}$ On electron microscopy the cells from these tumours show features of smooth muscle. ${ }^{2}$ The glomus is encompassed by non-myelinated nerve fibres. Glomera are present in greatest abundance in the skin of the distal parts of the limbs, but are widely distributed throughout the body. The glomus tumour must not be confused with the glomus jugulare tumour, also called the chemodectoma or nonchromaffin paraganglioma, which arises from the glomus jugulare situated in the dome of the jugular bulb in the depths of the upper portion of the neck. ${ }^{3}$

Glomus tumours are small and well defined and are seldom more than $4 \mathrm{~cm}$ in diameter. They are usually single but may be multiple, in which case there is sometimes a hereditary tendency. ${ }^{4}$ Occurring at all ages, they sometimes affect children. They are found most commonly in the skin of the peripheral portions of the limbs, and a favourite site is under a finger-nail. But they have been reported on the penis and the face, in the muscles and deeper tissues, and even in the stomach. ${ }^{5}$ A characteristic clinical feature is severe paroxysmal pain. Histologically the tumour is composed of vascular spaces, the walls of which contain even layers or irregular masses of uniform, cuboidal glomus cells with central, rounded, darkly-staining nuclei.

Recently W. F. Mullis and his colleagues have reviewed 35 cases of glomus tumour that were treated at the Hospital of the University of Pennsylvania between 1939 and $1971 .^{6}$ The patients' ages were between 15 and 68 years, and there was a slight predominance of women. Twenty-four of the tumours occurred on the upper limb, 13 being subungual and seven located on the forearm. Twelve of the 13 subungual tumours occurred in women, while all the forearm tumours were found in men. There were eight cases of glomus tumour on the lower limb, and none was subungual. Most of the lesions had a reddish-blue appearance, but a third were pale or neutral in colour. Three tumours were not located on the limbs: one was on the skin of the abdomen, one on the nose, and one in the stomach, where it led to massive gastrointestinal bleeding. All the tumours were painful except for those in the stomach and on the nose and one on the hip. One tumour was situated in the wrist joint, where it caused much discomfort.

The aetiology of the glomus tumour is unknown. In a few cases there is a history of trauma, but this is probably incidental. The condition must always be remembered in the differential diagnosis of painful subcutaneous nodules. Other tumours that can also cause pain at these sites are the leiomyoma, neurilemmoma, neuroma, and angiolipoma, but none of these is characteristically subungual. ${ }^{6}$ The treat- ment of a glomus tumour consists of complete excision. Provided this is adequate there should be no recurrence, for the tumour has no malignant potentiality. Three of the 35 cases reviewed by Mullis and his colleagues had been previously operated upon, but none of the cases in their series required a further operation after excision at the university hospital. In subungual glomus tumours the whole nail should be removed.

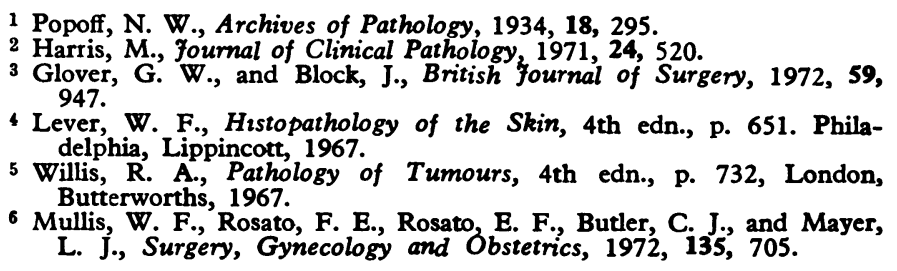

\section{Treatment of Spina Bifida Cystica}

The treatment of myelomeningoceles and hydrocephalus used to be a field for enthusiastic research among young neurosurgeons and a source of despondency among those who had already tried and failed. The development of the ventriculoatrial shunt (Holter and Pudenz valves especially) provided a treatment of hydrocephalus which was certainly more effective than anything hitherto and made the closure of myelomeningoceles appear to be worthwhile.

There were hopes of producing normal or almost normal children, whose only handicap was likely to be a defect of locomotion and probably incontinence. In several clinics every child born with spina bifida cystica was treated and the subsequent hydrocephalus likewise. In other clinics the treatment was carried out with the same enthusiasm, but with some selection, by omission of those with gross neurological deficit or multiple congenital abnormalities. Enormous numbers of children were treated, and there was serious concern about the provision of facilities for their subsequent education, care, and absorption into society. However, not only was there the expected need to revise the shunts and to carry out operations on the urinary tract and limbs, but the incidence of complications of all kinds was higher than had been expected. The quality of survival was less satisfactory than had been hoped, and many children spent a life of repeated hospital admissions or of semipermanent care in residential homes in which they learnt how to survive despite handicaps of daunting severity.

Disappointment in the results that were achieved led John Lorber, 1 who with his surgical colleagues in Sheffield had an experience which was far greater than most, to attempt to define prognostic criteria that could provide a basis for the selection of patients for treatment. A prospective study was initiated. It covered 201 infants with open myelomeningoceles born in 1967 and 1968, who were treated with all available skill from the first day of life. Initially, four criteria were thought to have a consistently grave prognosis: severe paraplegia, gross enlargement of the head, severe kyphosis, and associated gross congenital anomalies or major birth injuries. Retrospectively it was shown in addition that infants with thoracolumbar lesions, or a very severe degree of hydrocephalus at birth, had a particularly bad outlook. Not only was there considerable suffering for the severely disabled 
children-which included all those with adverse criteriabut grave problems beset the families and upset normal siblings. ${ }^{2}$

Having defined his adverse prognostic criteria, Lorber tested them against the patients admitted to the Sheffield unit during the last ten years. The results confirm their validity and raise the question of the moral justification for treating all patients with spina bifida cystica without some form of selection. As has been mentioned, all the patients with adverse criteria were severely disabled, but $59 \%$ died despite maximum efforts at treatment. On the other hand the survival of those with open myelomeningoceles but without the adverse criteria was $84 \%$, and their quality of survival was very much better. Half of them were of normal intelligence compared with only one-fifth of those with adverse criteria, who also required many more operations for their hydrocephalus and urinary and orthopaedic disturbances.

The problem of what treatment should be offered was presented in a particularly acute form at Hull recently. ${ }^{3}$ In that case the parents, who disagreed with their medical advisers, were overridden by the juvenile court on the application of the social services committee. Many people have regarded the circumstances of this case as disquieting.

It may be thought wrong to keep patients alive merely because it is technically possible to do so. A study by P. P. Rickham and T. Mawdsley ${ }^{4}$ shows that a miserable survival without treatment is not common, for they found only two children were alive after six months among 57 untreated cases of spina bifida cystica. Lorber has provided valuable evidence on which to base a system of selection. But there can be no universally applicable formula; every case must be regarded as an individual problem.

\footnotetext{
1 Lorber, J., Archives of Disease in Childhood, 1972, 47, 854.

2 Hare, E. H., Laurence, K. M., Payne, H., and Rawnsley, K., British Medical fournal, 1966, 2, 757

British Medical fournal, 1972, 4, 567.

- Rickham, P. P., and Mawdsley, T., Developmental Medicine and Child Neurology, 1966, 8, Supplement 1i, p. 20.
}

\section{Obesity and Coronary Heart Disease}

A particular type of person is often depicted as being specially liable to coronary artery disease. Characteristically he is an obese cigarette smoker, employed in a sedentary but stressful occupation, which affords him little physical activity, and he may have a family history of cardiovascular disease. On examination he may be hypertensive, have a corneal arcus, xanthelesma, and xanthomata, and the laboratory may detect hypercholesterolaemia, lipoprotein abnormalities, or diabetes mellitus.

How accurate is this profile? It is agreed that hypercholesterolaemia, cigarette smoking, hypertension, and diabetes are real risks, ${ }^{1-5}$ and it is generally accepted that obesity also promotes the development of coronary artery disease. ${ }^{7}$ This belief is based on detailed follow-up studies and on actuarial reports of insurance companies ${ }^{8-11}$ suggesting that there is a continuous direct relationship between body weight and coronary disease. However, the validity of this assumption has been questioned. 3467

Opinions are bound to conflict because we know so little about obesity. It lacks any absolute definition, and often no distinction is made between obesity and overweight. ${ }^{12}$ There is lack of agreement on how it can best be measured, and there are many methods of expressing body weight. There is even doubt about the accuracy of a measure which must estimate bone, muscle, and body shape in addition to obesity. ${ }^{710}$ Skinfold thickness is possibly a more accurate indicator than body weight of actual fatness, but measurements of it have not been adopted as a routine. ${ }^{12} 13$ Insurance companies' tables of desirable weight are based on the average weights of insurance applicants of given sex and height, aged about 25 , and arbitrarily divided into types of body frame to allow for skeletal variation. These tables have been much criticized. 467 Finally, epidemiological studies are subject to statistical difficulties, especially in evaluating the significance of obesity in isolation from other risk factors such as hypertension, hypercholesterolaemia, and cigarette smoking.

In an attempt to resolve some of these problems Ancel Keys and his colleagues have analysed the relationship of relative weight and skinfold thickness to the five-year incidence of coronary heart disease in 11,400 men aged 4059.14 There were three geographical groupings-northern European (Finland and the Netherlands), southern European (Italy and Yugoslavia), and U.S. railroad employees. Two measures of relative body weight were used, and skinfold thickness, which is independent of height, was also measured. A number of interesting findings emerge from this study. On the basis of relative body weight a greater proportion of the Americans (29.5\%) and southern Europeans $(23.1 \%)$ were overweight than were the northern Europeans $(13 \%)$. At first it appeared that those who were overweight (as judged by relative body weight) and those who were obese (as judged by measurement of skinfold thickness) among the Americans and southern Europeans were more liable than average to coronary artery disease, but this association did not approach significance in relation to death from this disease or to definite myocardial infarction. Multivariate analysis of the data showed that neither relative weight nor obesity was in itself a statistically significant risk in relation to future coronary heart disease when age, blood pressure, serum cholesterol, and smoking were comparable. But, as expected, relative weight and fatness correlated with blood pressure and serum cholesterol, and there was an inverse correlation with smoking habit, the smokers being thinner and lighter than the non-smokers.

It may be true, as these findings suggest, that obesity itself does not necessarily increase the risk of coronary artery disease. How then, should the physician advise the overweight patient? The answer would seem to be that, though overweight may not be an independent risk factor, there are many compelling grounds for weight reduction. In the first place, a diseased heart should not be subjected to the unnecessary strain of moving an overweight body. Secondly, obesity is associated with the development of hypertension, hyperlipidaemia, and diabetes, ${ }^{19}$ all of which are aetiologically important in coronary heart disease, ${ }^{1-5}$ and weight reduction should be the first (and is often the most successful) treatment for these conditions. Apart from the cardiovascular consequences, obesity is apt to be associated with sedentary habits and skeletal and pulmonary complications. 9

1 Doyle, J. T., Dawber, T. R., Kannel, W. B., Kinch, S. H., and Kahn, H. A., fournal of the American Medical Association, 1964, 190,886 . 